\title{
Evaluation of the Influence of Extraction Conditions on the Isolation and Identification of Volatile Compounds from Cagaita (Eugenia dysenterica) Using HS-SPME/GC-MS
}

\author{
Mauro R. Silva, ${ }^{a}$ Gustavo H. Bueno, ${ }^{b}$ Raquel L. B. Araújo, ${ }^{a}$ Inayara C. A. Lacerda, ${ }^{a}$ \\ Lucas G. Freitas, ${ }^{a}$ Harriman A. Morais, ${ }^{c}$ Rodinei Augusti ${ }^{\circledR d}$ and Júlio O. F. Melo ${ }^{\circledR *, b}$ \\ ${ }^{a}$ Departamento de Alimentos, Universidade Federal de Minas Gerais (UFMG), \\ 31270-901 Belo Horizonte-MG, Brazil \\ ${ }^{b}$ Departamento de Ciências Exatas e Biológicas, \\ Universidade Federal de São João Del-Rei (UFSJ), 35701-970 Sete Lagoas-MG, Brazil \\ 'Departamento de Ciências Básicas, Faculdade de Ciências Biológicas e da Saúde (FCBS), \\ Universidade Federal dos Vales do Jequitinhonha e Mucuri (UFVJM), \\ 39100-000 Diamantina-MG, Brazil \\ ${ }^{d}$ Departamento de Química, Universidade Federal de Minas Gerais (UFMG), \\ 31270-901 Belo Horizonte-MG, Brazil
}

\begin{abstract}
The objective of this study was to explore the extraction and identification of volatile organic compounds (VOCs) present in cagaita fruit (Eugenia dysenterica). Parameters such as type of extraction fiber, agitation, extraction time and extraction temperature were investigated. Initially, the VOCs were extracted using headspace solid-phase microextraction. Then, the compounds were identified using gas chromatography coupled to mass spectrometry. Results revealed the presence of at least 26 different compounds and the polyacrylate (PA) fiber promoted the extraction of a larger number of VOCs. Regarding the PA fiber, the most efficient extraction was achieved using a $50 \mathrm{rpm}$ agitation at $45{ }^{\circ} \mathrm{C}$ for $30 \mathrm{~min}$. The majority were monoterpenes $(34.64 \%)$ and esters $(36.28 \%)$. An increase in the extraction temperature promoted the isolation of more VOCs when using carboxen/polydimethylsiloxane (CAR/PDMS) and divinylbenzene/carboxen/polydimethylsiloxane (DVB/CAR/PDMS) fibers. However, these fibers allowed the isolation of a smaller number of VOCs. The extraction time and agitation had no significant influence.
\end{abstract}

Keywords: cagaita, HS-SPME, GC-MS, volatile organic compounds

\section{Introduction}

The Cerrado, which covers $22 \%$ of Brazil's territory, is the second largest biome in Latin America, only behind the Amazonian forest, and it is a source of many plant species that play an important role in folk medicine and in food. In this biome, there are different types of fruits, with unique sensory and nutritional characteristics, which gives them economic, through the commercialization of derived products, and nutritional importance, by their consumption..$^{1-3}$

One of these fruits is the Eugenia dysenterica DC, an exotic fruit of the Myrtaceae family, which is popularly

*e-mail: onesiomelo@gmail.com known as cagaita. This fruit has received significant attention because it has features that make it ideal for use in its fresh form or in preparations such as jams, fruit jellies, juices, liqueurs and ice cream. ${ }^{3}$ These fruit-bearing plant species have peculiar characteristics, including their scents, flavors and colors, and high contents of phenolic compounds, flavonoids and tannins, besides the presence of saponins and terpenes. The characteristic aroma of fruits is determined by the kind of volatile substances they produce. These substances are typically carboxylic acids, alcohols, aldehydes, ketones, esters, phenylpropanoids and terpenes. ${ }^{4}$ Therefore, several studies have been conducted to identify volatile organic compounds (VOCs) present in fruits such as pitanga (Eugenia uniflora L.), ${ }^{5}$ cherry (Prunus avium), ${ }^{6}$ pineapple (Ananas comosus) ${ }^{7}$ strawberry and 
lemon guavas (Psidium cattleianum Sabine) ${ }^{8}$ araça-boi (Eugenia stipitata), ${ }^{9}$ mango (Mangifera indica L.), ${ }^{10}$ jaboticaba (Myrciaria jaboticaba) ${ }^{11}$ and apple (Malus domestica Borkh.). ${ }^{12}$

To better characterize these fruits, various techniques have been used to isolate VOCs, such as headspace solidphase microextraction (HS-SPME), ${ }^{13-15}$ headspace static, ${ }^{16}$ dynamic headspace, ${ }^{16}$ supercritical fluid extraction, ${ }^{16}$ purge and vapor, solid-phase extraction, and liquid-liquid extraction. ${ }^{16}$ Among these techniques, HS-SPME stands out due to its concentration of the volatile compounds, absence of solvent, ease of incorporating into gas chromatography (GC) and applicability to a wide variety of analytes. ${ }^{16}$

The development of VOCs extraction method by HS-SPME involves the assessment of many parameters like fiber type, agitation, time and temperature of the extraction. The fiber features are critical since each fiber has different polarities, polymeric coating thicknesses, all of which are important variables for extracting the largest amount of a broad range of VOCs with distinctive features. Use of agitation may influence the equilibrium time and number of VOCs extracted. ${ }^{13,15,17-19}$

Thus, the present study aimed to optimize the best conditions for the identification of maximum VOCs of cagaita pulp by analyzing various parameters, such as type of extraction fiber, agitation, temperature and time. Finally, the research will provide relevant information about the fresh fruit in order to establish identity and quality standards.

\section{Experimental}

\section{Materials}

Five types of HS-SPME fibers with distinct characteristics were used to optimize the extraction of VOCs from cagaita samples. The fibers were manufactured by Sigma-Aldrich (St. Louis, MO, USA). Therefore, the semipolar fibers carboxen/polydimethylsiloxane (CAR/PMDS, $75 \mu \mathrm{m})$, divinylbenzene/carboxen/polydimethylsiloxane (DVB/CAR/PDMS, 50/30 $\mu \mathrm{m}$ ) and polydimethylsiloxane/ divinylbenzene (PDMS/DVB, $65 \mu \mathrm{m}$ ) and the polar fibers carbowax/divinylbenzene (CW/DVB, $65 \mu \mathrm{m})$ and polyacrylate $(\mathrm{PA}, 85 \mu \mathrm{m})$ were evaluated.

\section{Methods}

\section{Preparation of samples of cagaita}

The ripe fruit of cagaita were collected at Sete Lagoas, Minas Gerais State, Brazil (latitude 19²8’36” and longitude $\left.44^{\circ} 11^{\prime} 43^{\prime \prime}\right)$, in 2016 . Then, the fruit were transported to the laboratory of the Federal University of São João Del-Rey, Sete Lagoas-MG. The sample was washed in running water and then sanitized using $500 \mathrm{~mL}$ of sodium hypochlorite solution (200 ppm) for $15 \mathrm{~min}$ followed by a subsequent rinse under running water for $2 \mathrm{~min}$. After that, the seeds were discarded, and the pulp was homogenized using a mixer (Arno, São Paulo, Brazil). The homogenized pulp was stored at $-18^{\circ} \mathrm{C}$ until further use.

\section{Extraction of volatile compounds}

The extraction of VOCs was performed using SPME fibers (CAR/PMDS, DVB/CAR/PDMS, PDMS/DVB, CW/DVB and PA). For this process, $2.0 \mathrm{~g}$ samples of cagaita pulp were transferred to $20 \mathrm{~mL}$ vials, which were sealed with aluminium and rubber septa. Then, the vials were placed in an aluminium heating block $(8.5 \times 10 \mathrm{~cm})$ with a hotplate, and the same fibers for adsorption of volatile substances were inserted into each of them. The optimization of extraction conditions (sample stirring time, temperature, and extraction time) was conducted based on the procedure described by Rodrigues and Iemma. ${ }^{20}$ To this end, a $2^{3}$ full factorial design with triplicates at the central point was used, and the dependent variables were the extraction time $(20,30$ and $40 \mathrm{~min})$, the extraction temperature $\left(25,45\right.$ and $\left.65^{\circ} \mathrm{C}\right)$ and agitation (0,50 and $100 \mathrm{rpm})$. The number of volatile substances isolated for each test was used as response for the parameters evaluated.

After the adsorption of VOCs by the HS-SPME fibers, the fibers were directly introduced into the gas chromatograph injector, occurring desorption and separation of compounds in the chromatographic column, ionized by electron impact (EI) and finally identified by the mass analyzer.

\section{Identification of volatile compounds}

VOCs were identified in the Laboratory of Mass Spectrometry of the Department of Chemistry (UFMG), using a gas chromatograph (Trace GC Ultra) coupled to a mass spectrometer (MS, Polaris Q, Thermo Scientific, San Jose, CA, USA), with an ion trap, using a split/ splitless capillary injector. The chromatographic analysis was performed as described by Belo et al. ${ }^{21}$ Therefore, the following conditions were used: injector temperature of $250{ }^{\circ} \mathrm{C}, 5 \mathrm{~min}$ desorption, ion source temperature of $200{ }^{\circ} \mathrm{C}$ and $275{ }^{\circ} \mathrm{C}$ interface. Helium was used as the carrier gas at a flow rate of $1 \mathrm{~mL} \mathrm{~min}^{-1}$, and the VOCs were separated using an HP-5ms (5\% phenyl and 95\% methylpolysiloxane) capillary column $(30 \mathrm{~m} \times 0.25 \mathrm{~mm} \times 0.25 \mu \mathrm{m}$; Agilent Technologies Inc., Munich, Germany). Initially, the column 
was held at $40^{\circ} \mathrm{C}$ for $5 \mathrm{~min}$, and then, the temperature was increased at a rate of $2.5^{\circ} \mathrm{C} \mathrm{min}-1$ up to $125^{\circ} \mathrm{C}$ followed by an increase of $10^{\circ} \mathrm{C} \mathrm{min}^{-1}$ up to $245^{\circ} \mathrm{C}$ and held for $3 \mathrm{~min}$.

The acquisition of the data occurred in full scan mode with a range of $\mathrm{m} / \mathrm{z} 50$ to 350 , ionization by EI, and a power of $70 \mathrm{eV}$.

The VOCs were identified based on the comparison between mass spectrum obtained and the database available in library of the National Institute of Standards and Technology (NIST). In addition, the data were confirmed through comparison with compounds previously reported in the literature.

\section{Results and Discussion}

\section{Optimization of extraction conditions using HS-SPME}

The optimization of VOCs extraction conditions by HS-SPME were performed through multivariate analysis seeking to evaluate the effect of the agitation, time and temperature of extraction on each type of SPME fiber. The number of VOCs identified was considered as response, since this study aimed to optimize a method to determine the largest amount of volatile compounds in cagaita.

In Table 1, it is presented the relative area (\%) of the volatile compounds isolated using the fiber PA in the best response assay. When using the PA fiber, the best experimental condition was obtained by subjecting cagaita samples to $50 \mathrm{rpm}$ agitation at $45{ }^{\circ} \mathrm{C}$ for $30 \mathrm{~min}$. The greatest part of VOCs identified was monoterpene (34.64\%) and esters (36.28\%).

Table 1. Relative composition of volatile organic compounds (VOCs) identified in the cagaita using the polyacrylate (PA) fiber

\begin{tabular}{lcc}
\hline VOCs & Class & Area / \% \\
\hline 3-Buten-2-one & ketone & 2.04 \\
Oleic alcohol & alcohol & 9.83 \\
Ethyl acetate & ester & 5.53 \\
Dibutyl phthalate & ester & 25.05 \\
(Z)-9-Methyl octadecenoate & ester & 5.70 \\
Eucalyptol & monoterpene & 20.56 \\
$\alpha$-Terpinene & monoterpene & 14.08 \\
Propanoic acid & carboxylic acid & 1.16 \\
Nonanoic acid & carboxylic acid & 4.49 \\
Tetradecanoic acid & carboxylic acid & 1.77 \\
1-Methyl-4-(1-methylethyl)- & hydrocarbon & 1.65 \\
1,3-cyclohexadiene & & \\
2-Methyl-1,3-butadiene & hydrocarbon & 2.55 \\
Estragole & ether & 5.59 \\
\hline
\end{tabular}

García et al..$^{22}$ has found a different result when Barbados Cherry 'BRS-366 Jaburú' samples were subjected to a temperature of $65^{\circ} \mathrm{C}$ for $20 \mathrm{~min}$, using the PA fiber. Under these conditions, predominant extracted compounds were terpenes and carboxylic acids. These different results are associated with VOCs profile, which is characteristic for each matrix evaluated.

Effect of some parameters on the number of VOCs extracted

To investigate the influence of some parameters (agitation, time and temperature) on the extraction and subsequent identification of VOCs using each fiber type, Pareto diagrams were constructed with a $95 \%$ confidence limit, as shown in Figure 1.

As seen in Figure 1, the extraction behavior varied between the parameters tested for each type of fiber. Extraction temperature showed a significant effect only with CAR/PDMS and DVB/CAR/PDMS fibers. An increase in temperature with these two fibers allowed greater extraction and consequently the identification of a larger number of compounds. Each type of coating extracts different groups of VOCs. In that way, PDMS coating has affinity with non-polar compounds such as esters and monoterpenes, which are predominant in the samples of this study. These results are in agreement with those observed by Belo et al., ${ }^{21}$ who observed predominance of non-polar VOCs (esters and terpenoids) in pequi samples.

Agitation and time of extraction did not have a significant effect with any of the evaluated fibers. It was not found previous studies evaluating chemometric tools to optimize the extraction and identification of VOCs in cagaita using different fiber coatings.

\section{Effect of using different types of HS-SPME fiber}

The influence of the fiber type on the number of VOCs extracted from cagaita fruit is shown in Table 2. This table shows the total number of compounds found in the 11 assays of factorial planning for each fiber.

Based on the number of isolated compounds from each set of experimental conditions, PA fiber was the most efficient $(\mathrm{n}=17)$, followed by CAR/PDMS $(\mathrm{n}=16)$, PDMS/DVB $(n=15)$ and DVB/CAR/PDMS $(n=14)$. The CW/DVB fiber was the least effective, allowing isolation of only 12 compounds. It was observed that the PA polar fiber promoted the extraction of several compounds like alcohol, esters, ether, quinones, being efficient even for isolation of some nonpolar substances such as hydrocarbons shown in Table 2. These results highlight the importance of the use of different fibers and adsorption conditions in HS-SPME 


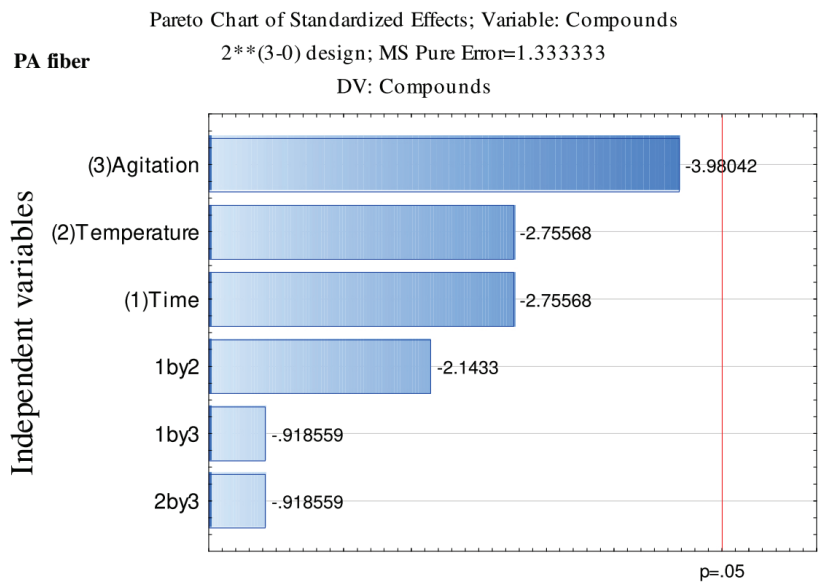

Standardized Effect Estimate (Absolute Value)

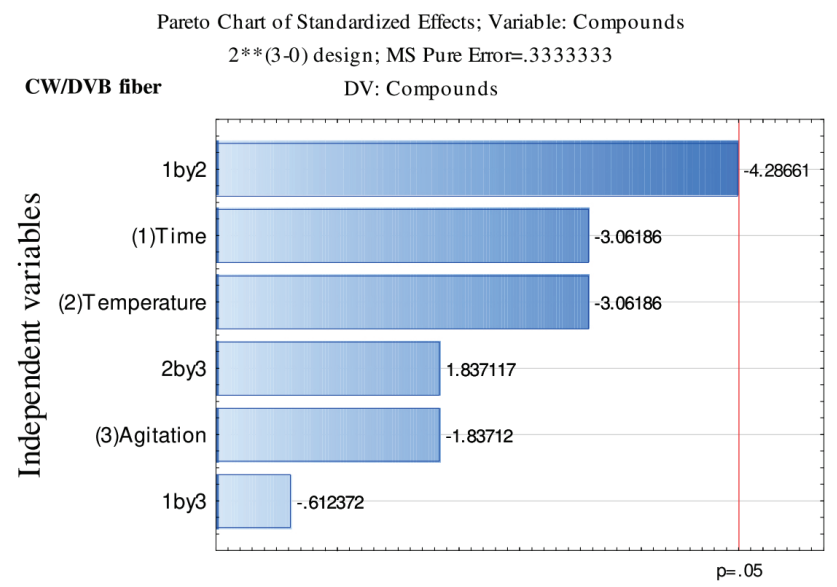

Standardized Effect Estimate (Absolute Value)

Pareto Chart of Standardized Effects; Variable: Compounds $2^{* *}(3-0)$ design; MS Pure Error $=, 3333333$

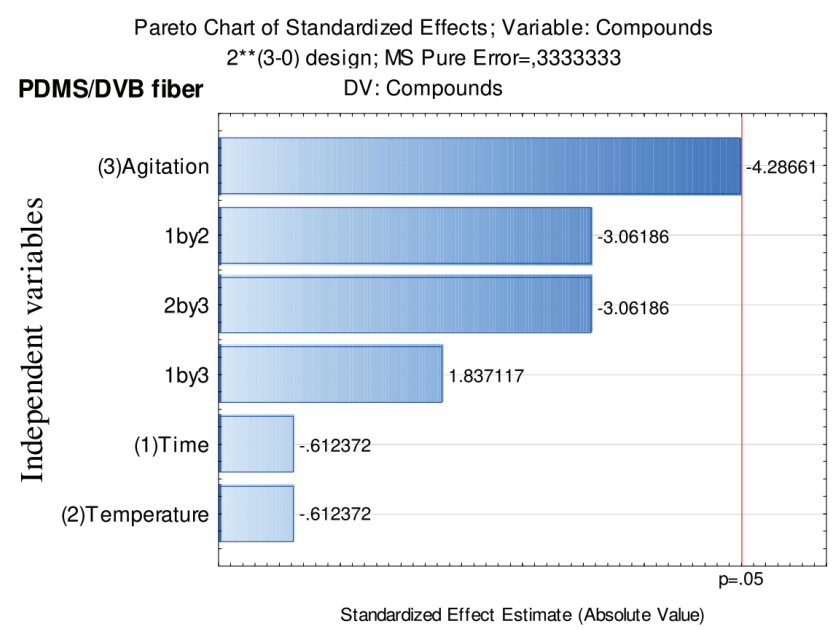

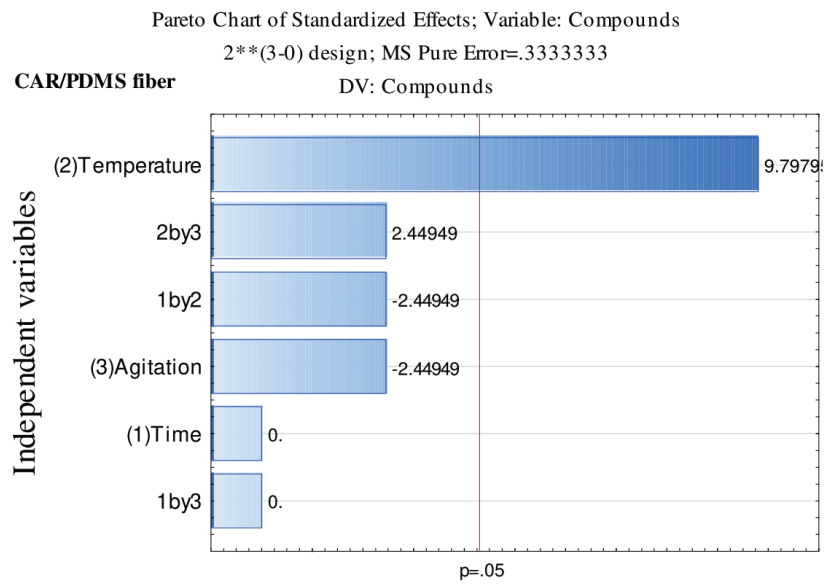

Standardized Effect Estimate (Absolute Value)

Pareto Chart of Standardized Effects; Variable: Compounds $2 * *(3-0)$ design; MS Pure Error $=1.333333$

DVB/CAR/PDMS fiber DV: Compounds

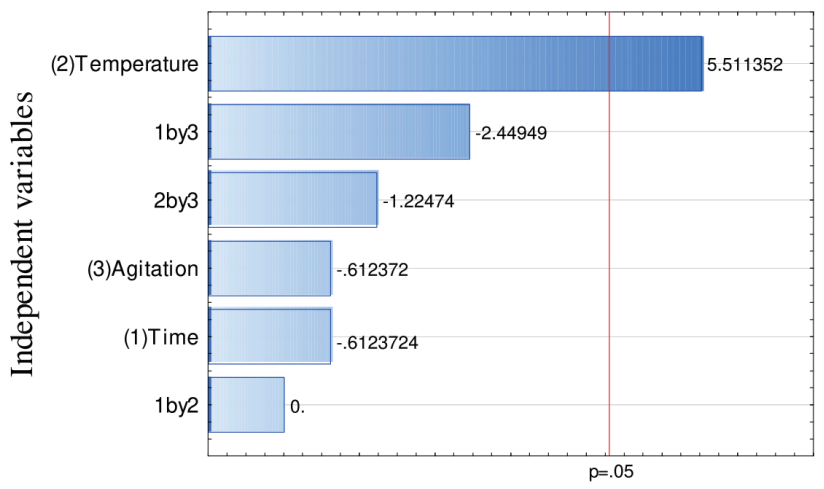

Standardized Effect Estimate (Absolute Value)

Figure 1. Effects of extraction parameters (agitation, time and temperature) on the isolation of VOCs from cagaita using different types of fibers.

to achieve a more complete identification of all volatile substances present in an extract.

No previous reports have evaluated the use of different types of fibers and HS-SPME to identify VOCs in cagaita. However, this type of study has previously been carried out for other types of fruits.
Sánchez-Palomo et al. ${ }^{17}$ evaluated three fibers with grape samples, and showed that the most effective fibers were a PDMS/DVB fiber, followed by a CAR/DVB/PDMS fiber and, finally, a CW/DVB fiber.

In the work of Pino and Quijano, ${ }^{23}$ the influence of three different fibers on the extraction of VOCs from plums 
Table 2. Volatile organic compounds (VOCs) identified from cagaita using different HS-SPME fibers

\begin{tabular}{|c|c|c|c|c|c|c|c|}
\hline \multirow{2}{*}{ No. } & \multirow{2}{*}{ VOCs } & \multirow{2}{*}{ class } & \multicolumn{5}{|c|}{ SPME fiber } \\
\hline & & & CAR/PDMS & CW/DVB & DVB/CAR/PDMS & $\mathrm{PA}$ & PDMS/DVB \\
\hline 1 & 3-buten-2-one & ketone & $x$ & $x$ & $x$ & $x$ & \\
\hline 2 & oleyl alcohol & alcohol & $x$ & $x$ & $x$ & $x$ & $x$ \\
\hline 3 & 2-propyn-1-ol & alcohol & & & & $x$ & \\
\hline 4 & ethyl acetate & ester & $x$ & $x$ & $x$ & $x$ & $x$ \\
\hline 5 & isopropyl myristate & ester & $x$ & & & & \\
\hline 6 & dibutyl phthalate & ester & $x$ & $x$ & $x$ & $x$ & $x$ \\
\hline 7 & (Z)-9-methyl octadecenoate & ester & $x$ & & & $x$ & $x$ \\
\hline 8 & 3-methyl acetate-1-butanol & ester & $x$ & & $x$ & & $x$ \\
\hline 9 & ethyl hexenoate & ester & & $x$ & & & \\
\hline 10 & eucalyptol & monoterpene & $x$ & $x$ & $x$ & $x$ & $x$ \\
\hline 11 & terpinolene & monoterpene & & & & & $x$ \\
\hline 12 & $\alpha$-terpinene & monoterpene & $x$ & $x$ & $x$ & $x$ & $x$ \\
\hline 13 & propanoic acid & carboxylic acid & $x$ & $x$ & $x$ & $x$ & \\
\hline 14 & heptanoic acid & carboxylic acid & & & & & $x$ \\
\hline 15 & nonanoic acid & carboxylic acid & $x$ & $x$ & $x$ & & $x$ \\
\hline 16 & undecylenic acid & carboxylic acid & $x$ & $x$ & & & \\
\hline 17 & dodecanoic acid & carboxylic acid & $x$ & & $x$ & $x$ & $x$ \\
\hline 18 & tridecanoic acid & carboxylic acid & & & & & $x$ \\
\hline 19 & tetradecanoic acid & carboxylic acid & $x$ & $x$ & $x$ & $x$ & $x$ \\
\hline 20 & 1,3-butadiene, 2-methyl & hydrocarbon & & & $x$ & & \\
\hline 21 & 1,3-diethyl-benzene & hydrocarbon & & & & $x$ & \\
\hline 22 & 2-methyl-1,3-butadiene & hydrocarbon & $x$ & $x$ & $x$ & $x$ & $x$ \\
\hline 23 & estragole & ether & $x$ & & $x$ & $x$ & \\
\hline 24 & 2,3-di-tert-butyl-p-benzoquinone & quinone & & & & $x$ & $x$ \\
\hline 25 & 2,4-bis-(1,1-dimethylethyl)-phenol & phenol & & & & $x$ & \\
\hline 26 & $\begin{array}{l}\text { 2-propen-1-one, 3-(4-methylphenyl)- } \\
\text { 1-phenyl-chalcone, 4-methyl }\end{array}$ & phenol & & & & $x$ & \\
\hline
\end{tabular}

HS-SPME: headspace solid-phase microextraction; CAR: carboxen; PDMS: polydimethylsiloxane; CW: carbowax; DVB: divinylbenzene; PA: polyacrylate.

was assessed, and the most efficient fiber was found to be a PDMS fiber, followed by a CAR/PDMS fiber, and a PDMS/DVB fiber being the least efficient. The consistency of these results in relation to the present study is worth noting; the CAR/PDMS fiber was more advantageous than the PDMS/DVB fiber.

In a third study, five types of fibers were employed by García et $a l .{ }^{24}$ for the adsorption of volatile substances present in acerola fruit that was obtained using a more efficient extraction process with a PA fiber, which allowed the identification of 37 compounds. In this work, the author noted that the least effective fiber was a CW/DVB fiber. Thus, these results are in agreement with the results obtained in the present study.

\section{Effect of temperature and extraction time}

The effects of temperature and extraction time on the number of VOCs extracted are presented in Figure 2.

It appears that the shorter the extraction time and higher the extraction temperature, the greater the number of VOCs identified. The shortest time $(20 \mathrm{~min})$ and the highest temperature $\left(65^{\circ} \mathrm{C}\right)$ were the best conditions for the adsorption of volatile substances when using the CAR/PDMS and DVB/CAR/PDMS fibers. This result shows that the use of longer times does not always enable greater detection of volatile substances depending on the fiber type. This may be related to the increased partition of volatile substances in the headspace, which increased the adsorption by the fibers. ${ }^{18,25}$ 

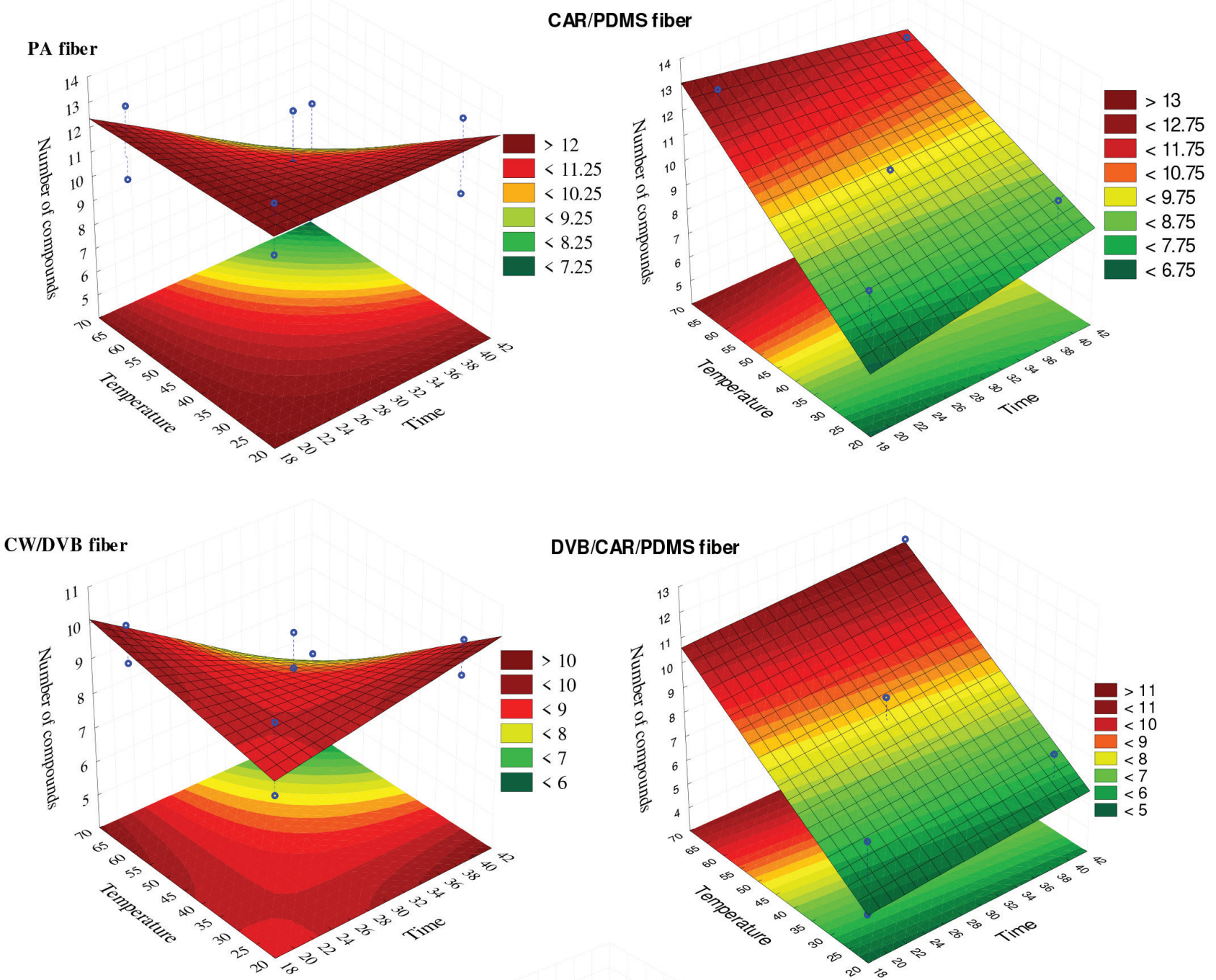

PDMS/DVB fiber

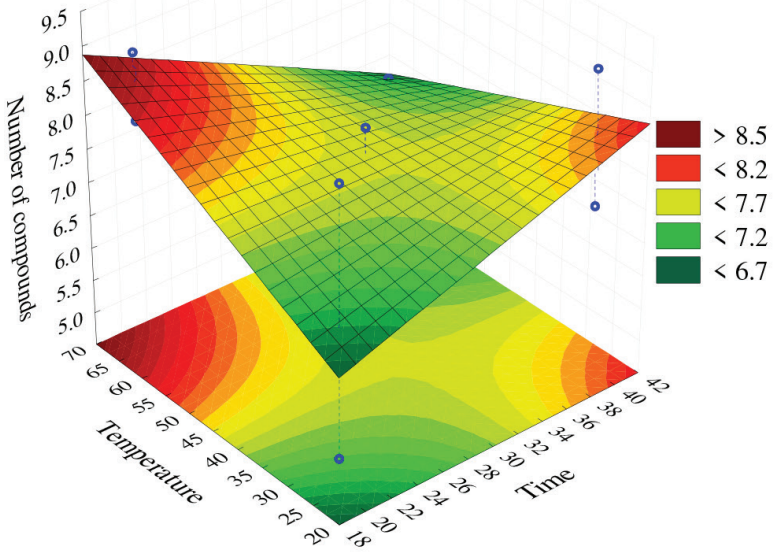

Figure 2. Effects of extraction temperature and time on the isolation of VOCs from cagaita using different types of fibers.

Considering that no previous reports have evaluated the effects of temperature and HS-SPME extraction time on VOCs of fruits grown in the Cerrado, the results obtained here were compared with previously reported results for grape samples, which were used in the only previous study that assessed this parameter. SánchezPalomo, ${ }^{17}$ who evaluated the optimal extraction conditions when employing a CAR/DVB/PDMS fiber, temperatures of 40 to $70{ }^{\circ} \mathrm{C}$, and times ranging from 10 to $50 \mathrm{~min}$, observed that the best results based on the sum of the 
peaks of aromatic compounds identified were obtained at $70{ }^{\circ} \mathrm{C}$ for $20 \mathrm{~min}$.

\section{Effects of agitation and extraction time}

Figure 3 shows the binomial agitation and time on the extraction of VOCs. As observed in the Pareto diagrams, agitation and time had no significant effect on extraction. In some fibers such as DVB/CAR/PDMS, the longer time and minimal or no agitation allowed the identification of the largest number of VOCs. It should be noted that no previous studies in the literature have addressed these two variables.

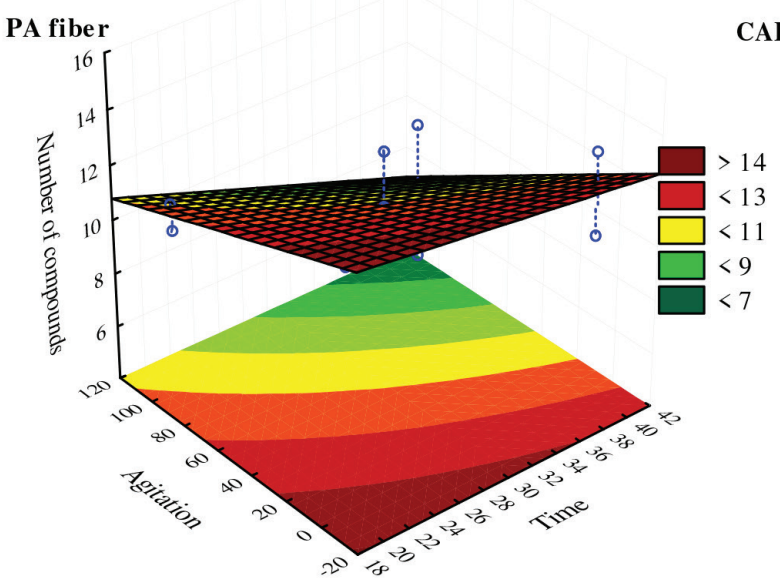

CAR/PDMS fiber

\section{CW/DVB fiber}

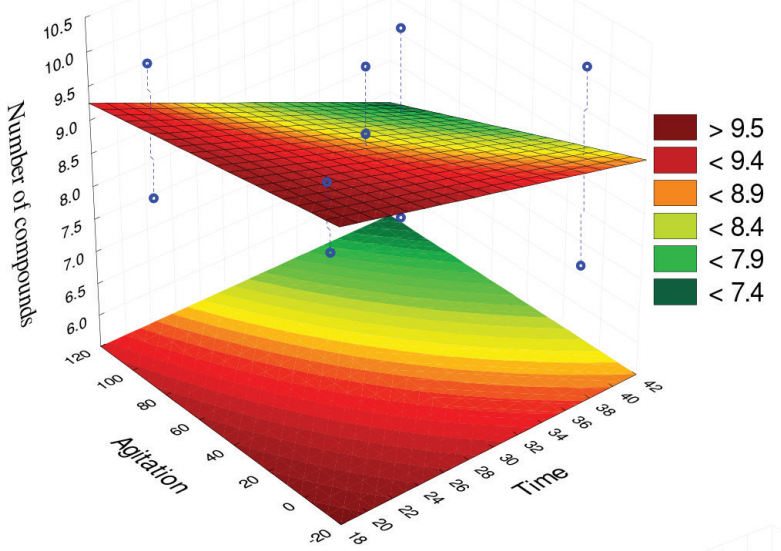

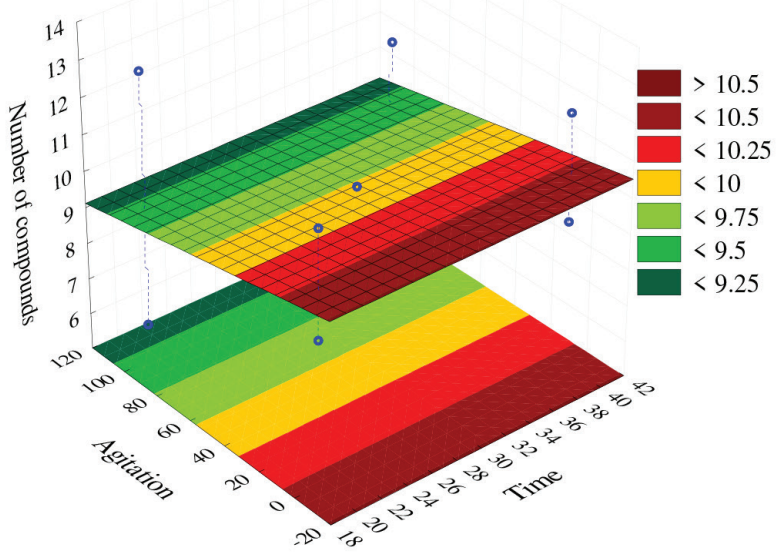

DVB/CAR/PDMS fiber

PDMS/DVB fiber
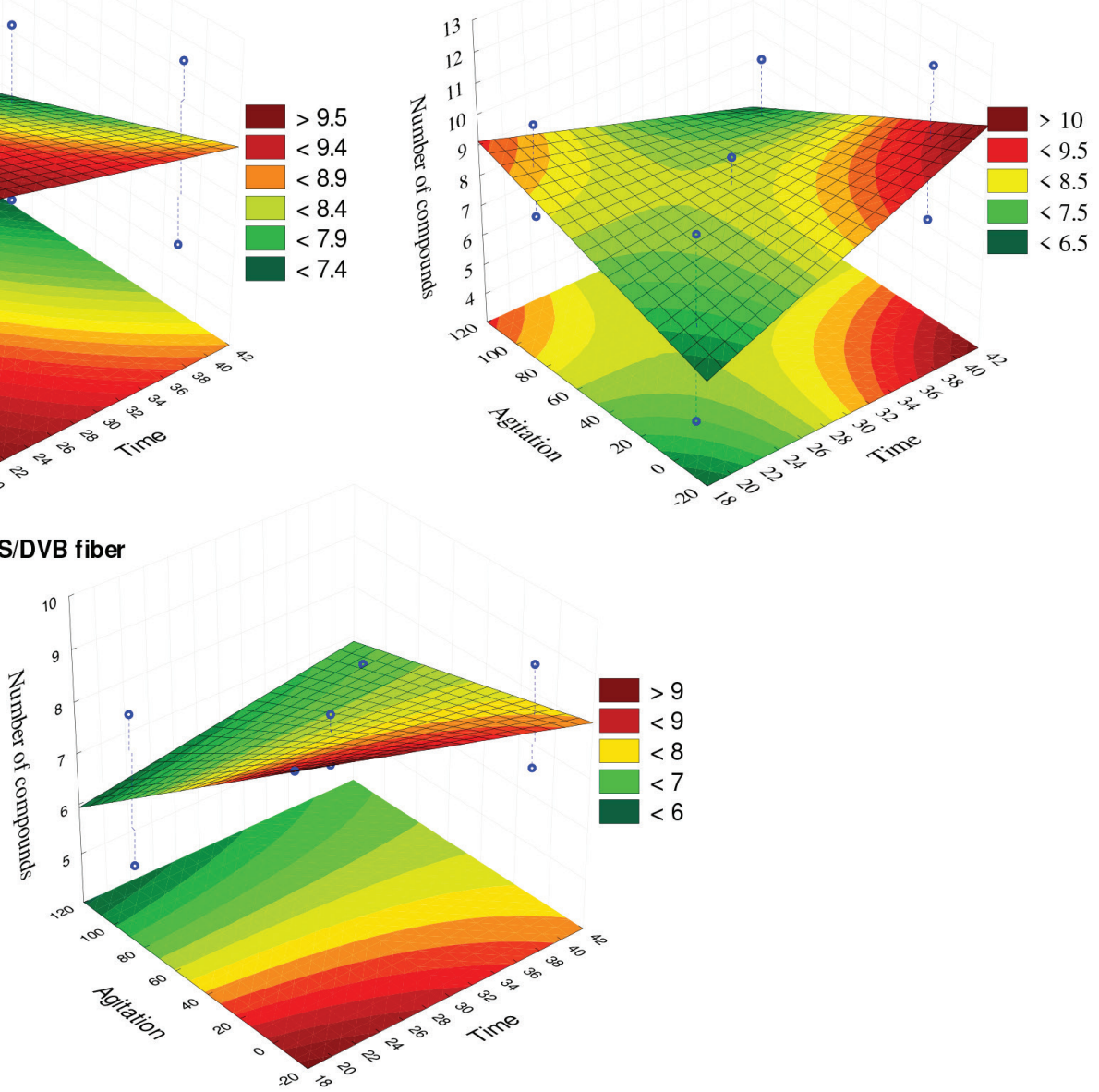

Figure 3. Effects of agitation and extraction time on the isolation of VOCs from cagaita using different types of fibers. 


\section{Effects of agitation and extraction temperature}

The effects of minimal agitation and higher extraction temperatures are shown in Figure 4. Note that these parameters had an influence on the number of VOCs only when CAR/PDMS and DVB/CAR/PDMS fibers were used
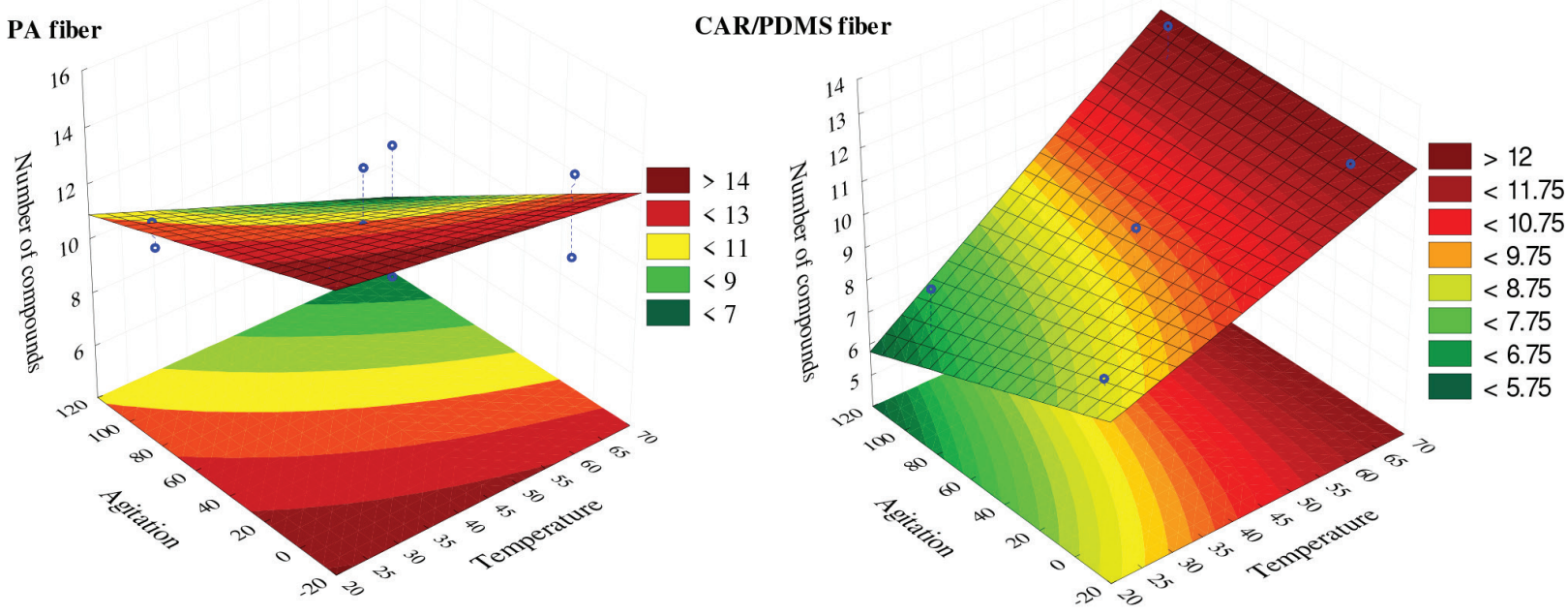

\section{CW/DVB fiber}

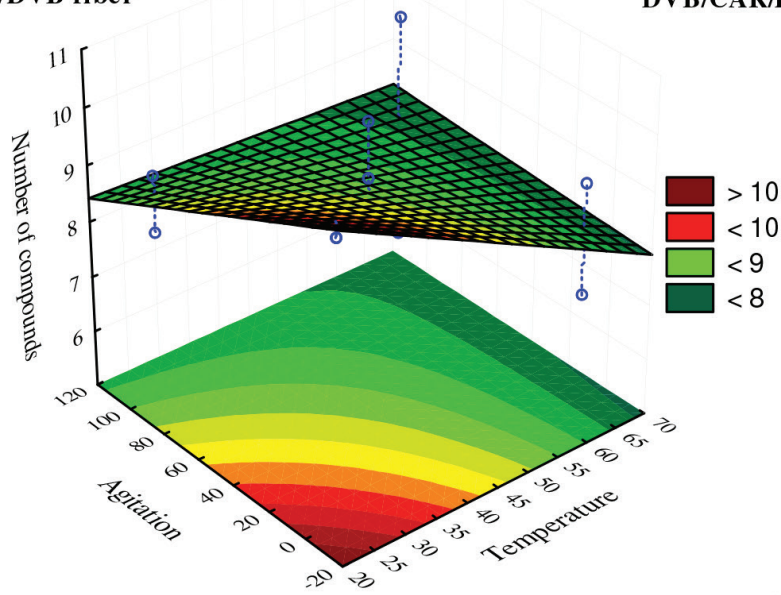

DVB/CAR/PDMS fiber

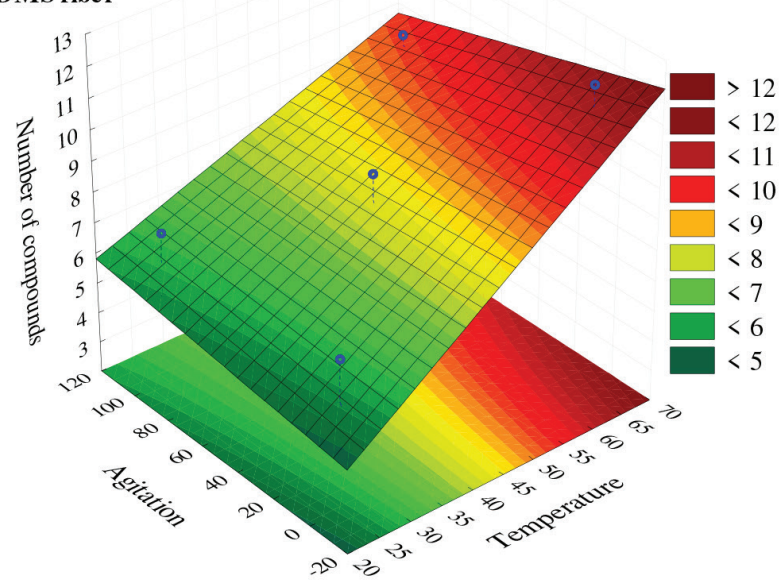

\section{PDMS/DVB fiber}

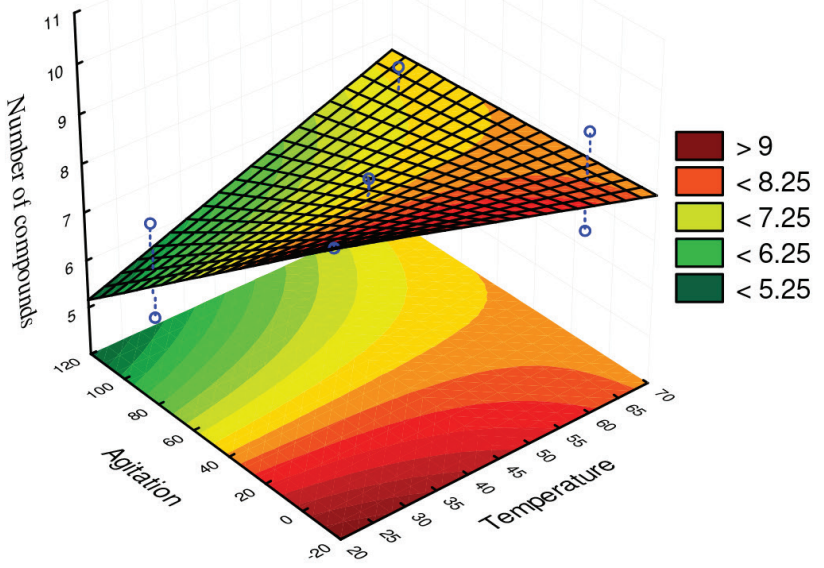

Figure 4. Effects of agitation and extraction temperature on the isolation of VOCs from cagaita using different types of fibers. 
and that minimal or no agitation and higher temperatures promoted the identification of a larger number of volatile substances. As observed previously, higher values of temperature are associated to a more efficient VOCs extraction.

It should be noted that this is the first report to address the dual effects of agitation and extraction temperature.

\section{Conclusions}

HS-SPME coupled to GC-MS has proven to be an efficient technique for the extraction and identification of the VOCs present in cagaita, allowing the identification of 26 compounds. The predominant substances were monoterpenes (34.64\%) and esters (36.28\%). The effects of the parameters studied were varied, and in terms of the number of compounds identified in all trials, PA fibers proved to be the most efficient. The use of higher temperatures allowed the identification of a larger number of VOCs, especially with CAR/PDMS and DVB/CAR/PDMS fibers. No significant effect of agitation and extraction time was observed.

\section{Supplementary Information}

Supplementary information (chromatograms and table of experimental design results) is available free of charge at http://jbcs.sbq.org.br as PDF file.

\section{Acknowledgments}

The authors thank CAPES and FAPEMIG for financial support.

\section{References}

1. Hashimoto, M. Y.; Costa, D. P.; Faria, M. T.; Ferreira, H. D.; Santos, S. C.; Paula, J. R.; Seraphin, J. C.; Ferri, P. H.; J. Braz. Chem. Soc. 2014, 25, 1504.

2. Cardoso, L. M.; Martino, H. S. D.; Moreira, A. V. B.; Ribeiro, S. M. R.; Pinheiro-Sant'ana, H. M.; Food Res. Int. 2011, 44, 2151.

3. Ribeiro, E. M. G.; Carvalho, L. M. J.; Ortiz, G. M. D.; Cardoso, F. S. N.; Viana, D. S.; Carvalho, J. L. V.; Gomes, P. B.; Tebaldi, N. M. In Food Industry; Muzzalupo, I., ed.; Viktorija Zgela: Rijeka, Croatia, 2013, ch. 1.

4. Bicas, J. L.; Molina, G.; Dionísio, A. P.; Barros, F. F. C.; Wagner, R.; Maróstica Jr., M. R.; Pastore, G. M.; Food Res. Int. 2011, 44, 1843.
5. Oliveira, A. L.; Lopes, R. B.; Cabral, F. A.; Eberlin, M. N.; Food Chem. 2006, 99, 1.

6. Vavoura, M. V.; Badeka, A. V.; Kontakos, S.; Kontominas, M. G.; Molecules 2015, 20, 1922.

7. Turazzi, F. C.; Morés, L.; Merib, J.; Carasek, E.; Narain, N.; Lima, L. K.; Nunes, M. L.; Food Sci. Technol. 2017, 37, 663.

8. Egea, M. B.; Pereira-Netto, A. B.; Cacho, J.; Ferreira, V.; Lopez, R.; Food Chem. 2014, 164, 272.

9. Franco, M. R. B.; Shibamoto, T.; J. Agric. Food Chem. 2000, 48, 1263.

10. Bonneau, A.; Boulanger, R.; Lebrun, M.; Maraval, I.; Gunata, Z.; Int. J. Food Sci. Technol. 2016, 51, 789.

11. Plagemann, I.; Krings, U.; Berger, R. G.; Marostica Jr., M. R.; J. Essent. Oil Res. 2012, 24, 45.

12. Giannetti, V.; Mariani, M. B.; Mannino, P.; Marini, F.; Food Control 2017, 78, 215.

13. Kataoka, H.; Lord, H. L.; Pawliszyn, J.; J. Chromatogr. A 2000, 880,35

14. Rocha, R. F. J.; Araújo, I. M. S.; Freitas, S. M.; Garruti, D. S.; J. Food Sci. Technol. 2017, 54, 4042.

15. Merkle, S.; Kleeberg, K. K.; Fritsche, J.; Chromatography 2015 , 2, 293.

16. Gutiérrez-Rosales, F. In Handbook of Fruit and Vegetable Flavors; Hui, Y. H., ed.; John Wiley \& Sons: New Jersey, USA, 2010, ch. 10.

17. Sánchez-Palomo, E.; Díaz-Maroto, M. C.; Pérez-Coello, M. S.; Talanta 2005, 66, 1152.

18. Abdullah, A. G. L.; Sulaiman, N. M.; Int. J. Food Eng. 2013, 9, 227.

19. Mesquita, P. R. R.; Nunes, E. C.; Santos, F. N.; Bastos, L. P.; Costa, M. A. P. C.; Rodrigues, F. M.; Andrade, J. B.; Microchem. J. 2017, 130, 79 .

20. Rodrigues, M. I.; Iemma, A. F.; Planejamento de Experimentos e Otimização de Processos, 2a ed.; Editora Casa do Pão: Campinas, Brazil, 2009.

21. Belo, R. F. C.; Augusti, R.; Lopes, P. S. N.; Junqueira, R. G.; Food Sci. Technol. 2013, 33, 116.

22. García, Y. M.; Guedes, M. N. S.; Rufini, J. C. M.; Souza, A. G.; Augusti, R.; Melo, J. O. F.; Sci. Electron. Arch. 2016, 9, 67.

23. Pino, J. Á.; Quijano, C. E.; Food Sci. Technol. 2012, 32, 76.

24. Garcia, Y. M. G.; Rufini, J. C. M.; Campos, M. P.; Guedes, M. N. S.; Augusti, R.; Melo, J. O. F.; J. Braz. Chem. Soc. 2019, $30,247$.

25. Setkova, L.; Risticevic, S.; Pawliszyn, J.; J. Chromatogr. A 2007, 1147, 213.

Submitted: July 11, 2018 Published online: September 21, 2018 